\title{
Distributed Control of UAV Formation Reconfiguration in Terms of Dynamic Reference Point
}

\author{
Guangyan $\mathrm{Xu}$, Dan Zhao, Jianchao Wang and Hongmei Zhang \\ Faculty of Aerospace Engineering, Shenyang Aerospace University, Shenyang, \\ China \\ guangyan_xu@163.com
}

\begin{abstract}
The design of a distributed controller in terms of DRP (dynamic reference point) to reconfigurate a formation of unmanned aerial vehicles (UAVs) is described. Reconfiguration of the UAV formation might be needed to avoid the obstacles and maintain the efficiency. Firstly, this paper proposes a concept of the DRP aiming at the problem of leader-follower UAV formation reconfiguration. Combining with the relative distance between the dynamic reference point and the leader in the coordinate, a new dynamic model of UAV formation reconfiguration is established and cleverly linearized. Secondly, a distributed formation reconfiguration controller in terms of DRP is designed based on the dynamic model. And the stability is also proved. The conventional controllers using distributed control law require the leader to be in front of the followers. However, the controller in terms of DRP designed in this paper doesn't limit the position relationships among UAVs. It is more useful and common. Finally, comparison between conventional general distributed controller and distributed controller based on DRP is simulated. The simulation results show that the controller designed in this paper is uniformly ultimately bounded and can complete formation reconfiguration task in realtime.
\end{abstract}

Keywords: Formation Reconfiguration, Dynamic Reference Point, Distributed Control

\section{Introduction}

Compared with a single drone, multiple UAVs formation has obvious advantages in the flexibility, fault tolerance and cooperation. Therefore, multiple UAV formation has been widely used in the area of search, environmental monitoring, coordinated operations and other civil or military fields. Formation control of multiple UAVs has also become a research hotspot in recent years.

The main purpose of the formation control is to coordinate and co-operate the multiple agents, making them to complete some task by the composition of certain formation. When the formation tasks or the environments change, UAV formation requires different formation control methods to complete formation reconfiguration. In the process of reconfiguration, we need to specify each drone in the new position on the team, and generate the trajectory which is from the original location to the new position. We have to ensure the flight safety as the premise and consider the dynamic characteristics of the aircraft and the input constraint conditions at the same time during generating the trajectory. Formation reconfiguration is one of the main research contents of formation control. The main control methods of formation control in common are leader -follower master-slave method, virtual structure method, and behavior based control method etc.[1]

M. Pachter et al proposed a formation flight controller design of autopilot based on aircraft flight in the literature [2] , which assumes that the self driving aircraft instrument equation is one order inertial link. And then a leader-follower formation 
controller was designed based on that. When the object of control oriented to actual formation, we must firstly complete the design of autopilot mentioned in this paper.

F. Zhao, Y. Wei and others, who are in Chengdu aircraft design and Research Institute and Northwestern Polytechnical University, used leader-follower model as the research model in the literature [3] , analyzing the aerodynamic coupling effects generated by the eddy current how to effect the wingman in all directions when the formation is in the tight flight and controlling the formation error using the control method, which can reach the ideal formation structure.

D. M. Wang, H. J. Fang et al, who are in Huazhong University of Science and Technology, established an adaptive control model that can select the virtual leader independently through the surroundings, by using the thoughts of predictive control in the literature [4] . It overcame the weaknesses and shortcomings of traditional leader-follower model whose information flow are one-way transfer. It also enhanced the robustness of the system. And what's more, it improved the stability of the whole formation system.

J. H. Reif et al used a potential field method which is similar to the behavior in [5] to avoid the individual members of formation becoming invalid due to their failures, and thus making the obtained sensor information transmission incomplete, so that this problem can be resolved smoothly.

J. R. Lawton et al in the literature [6] did some research about the method based on behavior, which made the deviation vector between each vehicle's true position and desired position value obtained consistency, and the direction of information flow is the ring topology of arbitrary direction, so as to realize the formation keeping.

W. Ren in [7] discussed the consistency of two order algorithm based on switch topology and the reference state, and put forward the control protocol of the three models.

W. W. Yu in [8], studied nonlinear multi-agent consensus problem of discrete time system, extended the definition of connected domain in the graph theory, put forward the control protocol based on graph theory and Lyapunov control theory, achieved asymptotically uniformly stability.

In practical application, the leader-follower formation model is widely used because it is simple and practical. But its anti-interference ability is not strong, especially if disturb the leader from outside, it will not work any more, and then the whole formation will be affected and even lead to the failure of the task execution. Therefore, this paper designs a UAV formation reconfiguration controller based on dynamic reference point. The controller can adjust the relative distance of the UAV to fast reconfigurate the formation and shorten the UAV team reconfiguration process time series during the UAV formation is in reconfiguration.

\section{Dynamic Model of UAV Formation In Terms of Dynamic Reference Point}

Considering a set of $\mathrm{n} U A V s$, whose kinematical equation is

$$
\begin{aligned}
& \dot{X}_{i}=v_{i} \cos \psi_{i} \\
& \dot{Y}_{i}=v_{i} \sin \psi_{i} \\
& \dot{\psi}_{i}=\omega_{i} \\
& \dot{v}_{i}=a_{i}
\end{aligned}
$$

where $X_{i} \in R$ and $Y_{i} \in R$ give the location of UAV in the inertia coordination; $v_{i}, \psi_{i}$ are flight speed and heading angular, respectively $; \omega_{i}, a_{i}$ are heading rate and acceleration respectively; $i=1,2 \cdots n$ is the index for the UAVs in the formation. In the formation 
dynamic model, state variables are $\left(X_{i}, Y_{i}, v_{i}, \psi_{i}\right)$, control variables are $\left(a_{i}, \omega_{i}\right)$. We can see that the model is a typical under actuated system.

For the UAV formation transformation problem, we can generally decouple the threedimensional motion model into two-dimensional plane motion and the vertical height of motion. Assuming the UAV can autonomously avoid collision motion control by adjusting the relative height of the plane; this paper takes the plane motion model as the study of formation control of UAV formation transformation.

The relationship of neighbor UAVs' location in the formation is shown in Fig.1.

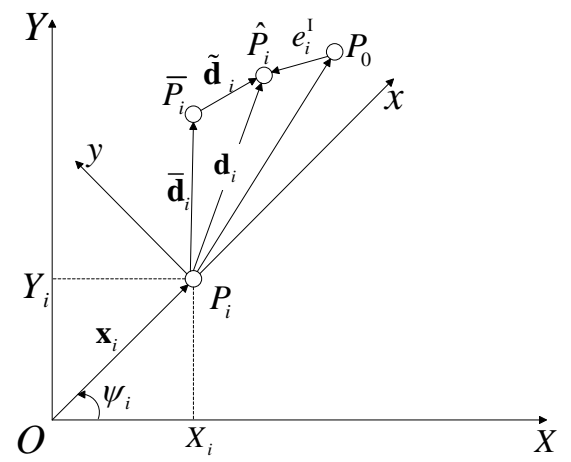

\section{Figure 1. Dynamic Reference Point of UAVs}

$P_{0}$ denotes the leader $\mathrm{UAV}, P_{i}$ denotes the $i$-th $\mathrm{UAV} ; \bar{P}_{i}$ and $\hat{P}_{i}$ are the desired reference point and the dynamic reference point of $P_{i}$, respectively. Among them, $\hat{P}_{i}$ is a time-varying variable. The formation parameters chosen in this paper are relative to the leader UAV, which can greatly reduce the error accumulation.

Introducing the non-linear transformation

$$
\mathbf{x}_{i}=\left(\begin{array}{c}
X_{i} \\
Y_{i}
\end{array}\right), \zeta_{i}=\left(\begin{array}{c}
v_{i} \cos \psi_{i} \\
v_{i} \sin \psi_{i}
\end{array}\right), \boldsymbol{u}_{i}=M_{i}^{-1}\left(\begin{array}{c}
a_{i} \\
\omega_{i}
\end{array}\right)
$$

where $\mathbf{x}_{i}, \zeta_{i}$ are the location and the velocity of the $i$-th UAV in inertial coordination; $\boldsymbol{u}_{i}$ is control input; transformation matrix $M_{i}$ is.

$$
M_{i}=\left(\begin{array}{cc}
\cos \psi_{i} & \sin \psi_{i} \\
-\frac{\sin \psi_{i}}{v_{i}} & \frac{\cos \psi_{i}}{v_{i}}
\end{array}\right)
$$

For the reason that the velocity of UAV will never be zero during the flight, $M_{i}$ is not a singular matrix. Then, the UAV kinematics equation (2) can be written as

$$
\begin{aligned}
& \dot{\mathbf{x}}_{i}=\zeta_{i} \\
& \dot{\zeta}_{i}=\boldsymbol{u}_{i}
\end{aligned}
$$

Introduce the expectation formation parameter $\overline{\mathbf{d}}_{i}$, which is the $i$-th UAV's relative distance with adjacent UAVs in the $x P_{i} y$ coordinate under the expectation reference speed.

$$
\overline{\mathbf{d}}_{i}=\left[\begin{array}{l}
\bar{d}_{i}^{x} \\
\bar{d}_{i}^{y}
\end{array}\right]
$$

$\overline{\mathbf{d}}_{i}$ is a constant vector. Furthermore, introduce a vector $\mathbf{d}_{i}$ to denote the $i$-th UAV's dynamic reference point in the $x P_{i} y$ coordinate

$$
\mathbf{d}_{i}=\left[\begin{array}{l}
d_{i}^{x}(t) \\
d_{i}^{y}(t)
\end{array}\right]
$$


$\mathbf{d}_{i}$ is a time-varying vector.

Error formation parameter $\tilde{\mathbf{d}}_{i}$ can be expressed as

Formula (7) can also be expressed as

$$
\tilde{\mathbf{d}}_{i}=\mathbf{d}_{i}-\overline{\mathbf{d}}_{i}
$$

$$
\mathbf{d}_{i}=\tilde{\mathbf{d}}_{i}+\overline{\mathbf{d}}_{i}
$$

Design an equation

$$
\dot{\tilde{\mathbf{d}}}_{i}=-K^{I I} \tilde{\mathbf{d}}_{i}
$$

The initial value of $\tilde{\mathbf{d}}_{i}$ can be determined by the formation parameters before the formation reconfiguration, it can be expressed as

$$
\tilde{\mathbf{d}}_{i 0}=\mathbf{d}_{i}\left(t_{0}\right)-\overline{\mathbf{d}}_{i}
$$

The value of $\tilde{\mathbf{d}}_{i}$ can tend to zero, $\tilde{\mathbf{d}}_{i} \rightarrow 0$ if matrix $K^{I I}$ is suitable selected.

Now, define the position and velocity error vectors as

$$
\begin{aligned}
& \boldsymbol{e}_{i}^{I}=\left(\mathbf{x}_{i}+\boldsymbol{d}_{i}\right)-\mathbf{x}_{0}=\left(\mathbf{x}_{i}+\overline{\boldsymbol{d}}_{i}+\tilde{\boldsymbol{d}}_{i}\right)-\mathbf{x}_{0} \quad i=\forall 2, . ., n \\
& \boldsymbol{e}_{i}^{I I}=\zeta_{i}-\boldsymbol{\zeta}_{0}
\end{aligned}
$$

Comprehensive equation (4) and (9), we can get the derivation of (11)

$$
\begin{aligned}
& \dot{\boldsymbol{e}}_{i}^{I}=\boldsymbol{e}_{i}^{I I}-K^{I I} \tilde{\mathbf{d}}_{i} \\
& \dot{\boldsymbol{e}}_{i}^{I I}=\mathbf{u}_{i}-\mathbf{u}_{0} \\
& \dot{\tilde{\mathbf{d}}}_{i}=-K^{I I} \tilde{\mathbf{d}}_{i}
\end{aligned}
$$

Equation (12) can be written in matrix form

$$
\left(\begin{array}{c}
\dot{\mathbf{e}}_{i}^{I} \\
\dot{\mathbf{e}}_{i}^{I I} \\
\dot{\tilde{\mathbf{d}}}_{i}
\end{array}\right)=F\left(\begin{array}{c}
\mathbf{e}_{i}^{I} \\
\mathbf{e}_{i}^{I I} \\
\tilde{\mathbf{d}}_{i}
\end{array}\right)+M \mathbf{u}_{i}+N \mathbf{u}_{0}
$$

where

$$
F=\left(\begin{array}{ccc}
0 & I & -K^{I I} \\
0 & 0 & 0 \\
0 & 0 & -K^{I I}
\end{array}\right), M=\left(\begin{array}{l}
0 \\
I \\
0
\end{array}\right), N=\left(\begin{array}{c}
0 \\
-I \\
0
\end{array}\right)
$$

Let

$$
\mathbf{e}_{i}=\left(\begin{array}{c}
\mathbf{e}_{i}^{I} \\
\mathbf{e}_{i}^{I I} \\
\tilde{\mathbf{d}}_{i}
\end{array}\right)
$$

Then the equation (13) can be expressed as

$$
\dot{\mathbf{e}}_{i}=F \mathbf{e}_{i}+M \mathbf{u}_{i}+N \mathbf{u}_{0}
$$

where $\mathbf{u}_{0}$ is the control input of the leader $\mathrm{UAV}$ in the formation reconfiguration.

\section{Controller Design of UAV Formation Reconfiguration}

According to the formula(16), consider the feedback control law

$$
\mathbf{u}_{i}=-K \mathbf{e}_{i}
$$

where, the feedback gain $K$ is

$$
K=\left[\begin{array}{lll}
K^{I} & K^{I I} & 0
\end{array}\right]
$$

Substituted formula (15) and (18) into equation (17), and we can get formula (19)

$$
\mathbf{u}_{i}=-\left[\begin{array}{lll}
K^{I} & K^{I I I} & 0
\end{array}\right]\left(\begin{array}{c}
\mathbf{e}_{i}^{I} \\
\mathbf{e}_{i}^{I I} \\
\tilde{\mathbf{d}}_{i}
\end{array}\right)=-K^{I} \mathbf{e}_{i}^{I}-K^{I I I} \mathbf{e}_{i}^{I I}
$$


In the above formula, $\mathbf{e}_{i}^{I}$ and $\mathbf{e}_{i}^{I I}$ are errors of the state variables. In formula (19), simple candidates of matrices $K^{I}, K^{I I I}$ are

$$
K^{I}=k_{1} I, K^{I I I}=k_{3} I, k_{1}, k_{3} \in R
$$

According to (2), control law $\left(a_{i}, \omega_{i}\right)$ can be derived to

$$
\left(\begin{array}{c}
a_{i} \\
\omega_{i}
\end{array}\right)=-M_{i}\left(K^{I}\left(\left(\mathbf{x}_{i}-\mathbf{x}_{0}\right)+\overline{\boldsymbol{d}}_{i}+\tilde{\boldsymbol{d}}_{i}(t)\right)+K^{I I I}\left(\zeta_{i}-\zeta_{0}\right)\right)
$$

According to equation (21), when controlling the $i$-th UAV, the control law of the system depends on three elements: the $i$-th UAV's relative distance from the leader UAV, the $i$-th UAV's the speed from the leader UAV and a time-varying $\tilde{\mathbf{d}}_{i}(t)$, the $\tilde{\mathbf{d}}_{i}(t)$ can be solved as (22), it tends to zero if matrix $K^{I I}$ is suitable selected.

$$
\tilde{\mathbf{d}}_{i}(t)=\left(\begin{array}{cc}
e^{-\lambda_{1}\left(K^{I I}\right) t} & 0 \\
0 & e^{-\lambda_{2}\left(K^{I I}\right) t}
\end{array}\right) \tilde{\mathbf{d}}_{i}\left(t_{0}\right) \rightarrow 0
$$

\section{Stability Analysis}

According to the last section, using the controller (21), the error dynamics (16) can be derived and expressed to

$$
\dot{\mathbf{e}}_{i}=\left(\begin{array}{c}
\dot{\mathbf{e}}_{i}^{I} \\
\dot{\mathbf{e}}_{i}^{I I} \\
\dot{\tilde{\mathbf{d}}}_{i}
\end{array}\right)=W\left(\begin{array}{c}
\mathbf{e}_{i}^{I} \\
\mathbf{e}_{i}^{I I} \\
\tilde{\mathbf{d}}_{i}
\end{array}\right)+N \mathbf{u}_{0}
$$

In the formula (23), matrix $W$ is

$$
\begin{aligned}
& W=F-M\left[\begin{array}{lll}
K^{I} & K^{I I} & 0
\end{array}\right] \\
& =\left(\begin{array}{ccc}
0 & I & -K^{I I} \\
0 & 0 & 0 \\
0 & 0 & -K^{I I}
\end{array}\right)-\left(\begin{array}{l}
0 \\
I \\
0
\end{array}\right)\left[\begin{array}{lll}
K^{I} & K^{I I I} & 0
\end{array}\right] \\
& =\left(\begin{array}{ccc}
0 & I & -K^{I I} \\
-K^{I} & -K^{I I I} & 0 \\
0 & 0 & -K^{I I}
\end{array}\right)
\end{aligned}
$$

Theorem 1: Suppose that matrices $K^{I}, K^{I I}, K^{I I I}$ in (24) are

$$
K^{I}=k_{1} I, K^{I I}=k_{2} I, K^{I I I}=k_{3} I
$$

in which, all parameters $k_{1}, k_{2}, k_{3} \in R^{+}$are positive real numbers. Then matrix $\boldsymbol{W}$ in (23) is Hurwitz.

Proof: Considering (25), formula (24) becomes

$$
W=\left(\begin{array}{ccc}
0 & I & -k_{2} I \\
-k_{1} I & -k_{3} I & 0 \\
0 & 0 & -k_{2} I
\end{array}\right)
$$

The eigenvalues of matrix $\boldsymbol{W}$ can be solved as follows

$$
\begin{aligned}
& \lambda_{1,2}=-k_{2} \\
& \lambda_{3,4}=-\frac{k_{3}}{2}+\frac{\sqrt{k_{3}^{2}-4 k_{1}}}{2} \\
& \lambda_{5,6}=-\frac{k_{3}}{2}-\frac{\sqrt{k_{3}^{2}-4 k_{1}}}{2}
\end{aligned}
$$


Obviously, if a system is stable, all the eigenvalues of the state transition matrix to the system need to have negative real parts. If $\lambda_{1,2}<0$ clearly $k_{2}>0$; For if $\lambda_{5,6}<0$, just need $k_{3}>0$; Let $\lambda_{3,4}<0$, then $\sqrt{k_{3}^{2}-4 k_{1}}<k_{3}$, we can get $k_{1}>0$ by solving the inequality. Above all, seeing from the eigenvalues of matrix $\boldsymbol{W}$, if $k_{1}>0, k_{2}>0, k_{3}>0$, it will meet that all the eigenvalues $\lambda_{1,2}<0, \lambda_{3,4}<0, \lambda_{5,6}<0$. So in the formula (27), $k_{1}>0, k_{2}>0, k_{3}>0$. Thus by the conclusion of Theorem 1, matrix $\boldsymbol{W}$ is Hurwitz. And the system is asymptotically stable.

It is not difficult to prove that if matrix $\boldsymbol{W}$ is Hurwitz, the flight path of the leader UAV controlled by $\mathbf{u}_{0}$ is UUB for the formula (23).

According to the previous work, the position of the leader UAV is restrained. Leader UAV only can be in front of the followers. However, it just requires $k_{1}>0, k_{2}>0, k_{3}>0$ in this paper, there is no requirement about $\mathbf{d}_{i}$. It is indicated that the leader UAV can not only in front of the followers but also at the back of the followers.

Compared with the previous work, there is no velocity feedback. Nevertheless, from the above proof, it can be seen that $k_{3}>0$, which means the system must have velocity feedback.

\section{Simulations}

The simulation results on UAVs formation reconfiguration can be obtained from the Matlab tool. In this paper, we consider a 3-UAV formation reconfiguration in simulation. The simulation time is given as 50s. The whole process of formation flight of three aircraft is in the same plane. The desired formation velocity is $50 \mathrm{~m} / \mathrm{s}$. In order to avoid the obstacles, the UAVs fleet changes the formation from formation 1 to formation 2. And when the UAVs avoid the obstacles successfully, it turns back to formation 1 again. The formation 1's desired distances relative to the leader are $d_{12}(50,100), d_{13}(100,200)$, and the formation 2's desired distances relative to the leader are $d_{22}(50,0), d_{23}(100,0)$. Simulations are performed on the comparisons between DRP (Dynamic Reference Point) distributed controller and general distributed controller, validating the feature of distributed steady state control based on dynamic reference point for UAV formation reconfiguration. 


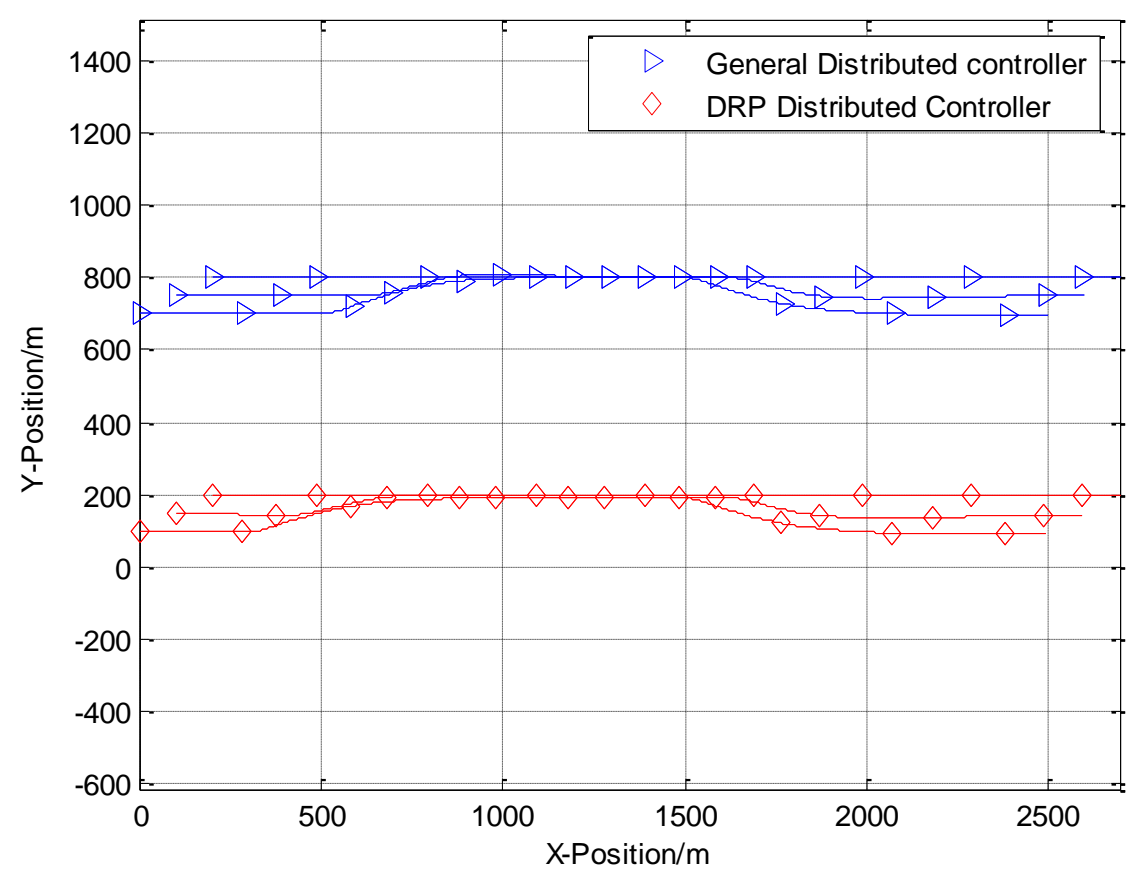

Figure 2. The Formation Performance between DRP Distributed Controller and General Distributed Controller $\left(v_{d}=50 \mathrm{~m} / \mathrm{S}\right)$

As shown in Figure 2, the formation flight performance with DRP distributed strategy and general distributed strategy. Apparently, the DRP distributed controller has the advantage in UAV formation reconfiguration for response speed and convergence speed. Furthermore, formation control with DRP distributed controller gains a good qualification with the desired distance. It also reveals that DRP distributed controller has a better control on the formation reconfiguration during the flight.

The comparison of control variant on angular velocity and acceleration between DRP distributed strategy and general distributed strategy is also simulated. In the simulation, the scopes of angular velocity and acceleration are shown as follows:

DRP Distributed Controller (red line):

UAV2 (-91.5 8.4, -1.66 0.755),

UAV3 (-171 112,-3.35 1.74);

General Distributed Controller (blue line):

UAV2 (-730 400, -8.2 14.62),

UAV3 (-1590 1222,-17.86 1.61);

For a better formation reconfiguration, DRP distributed strategy works at the early time. Therefore, the formation can be kept in less time than general distributed strategy. Also, the control cost is decreased in DRP distributed strategy. 


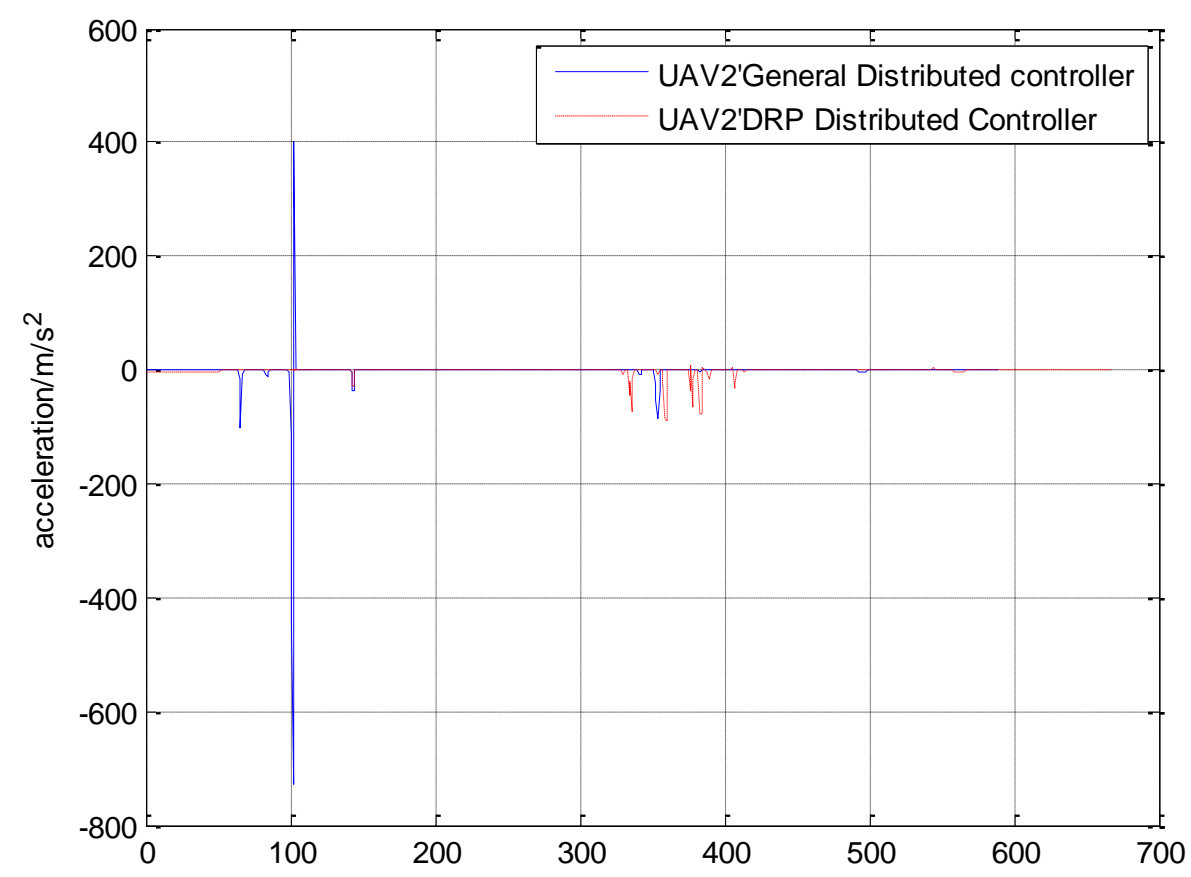

Figure 3. The Acceleration of UAV2 between DRP Distributed Controller and General Distributed Controller $\left(v_{d}=50 \mathrm{~m} / \mathrm{s}\right)$

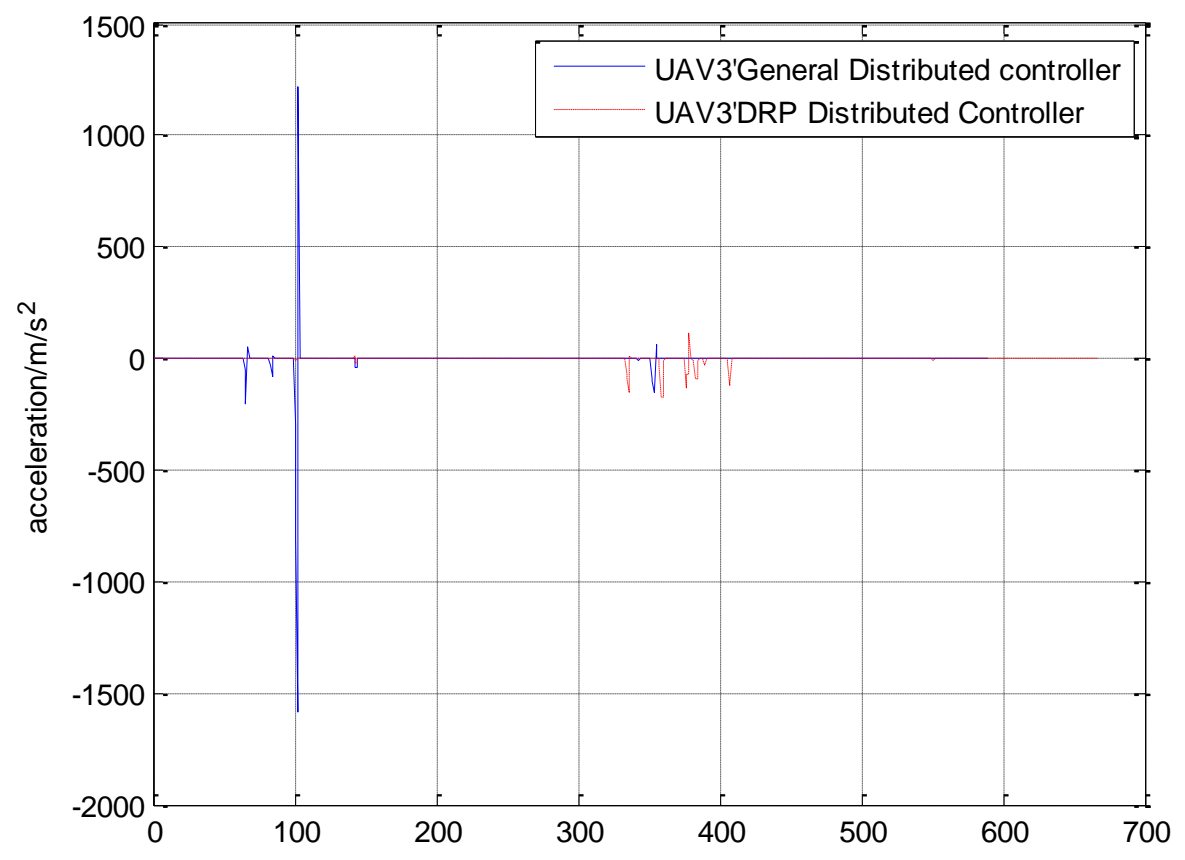

Figure 4. The Acceleration of UAV3 between DRP Distributed Controller and General Distributed Controller $\left(v_{d}=50 \mathrm{~m} / \mathrm{s}\right)$ 


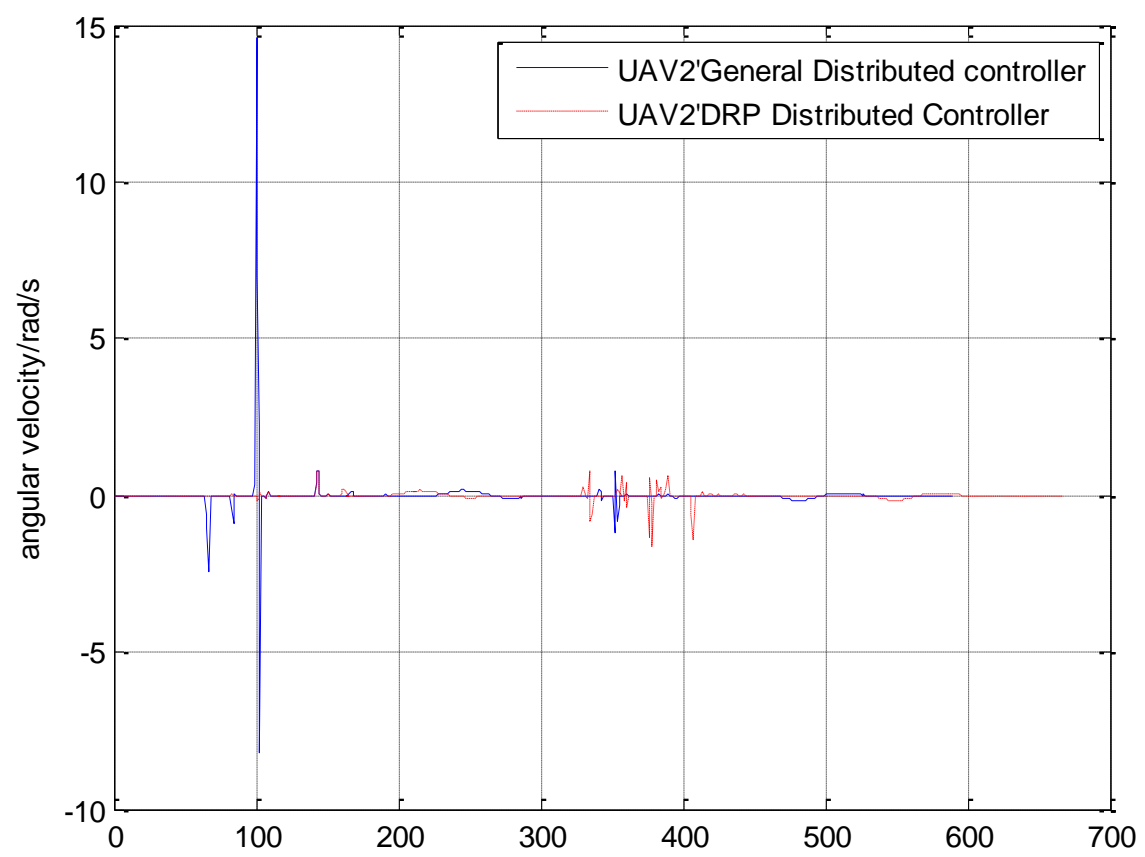

Figure 5. The Angular Velocity of UAV2 between DRP Distributed Controller and General Distributed Controller $\left(v_{d}=50 \mathrm{~m} / \mathrm{s}\right)$

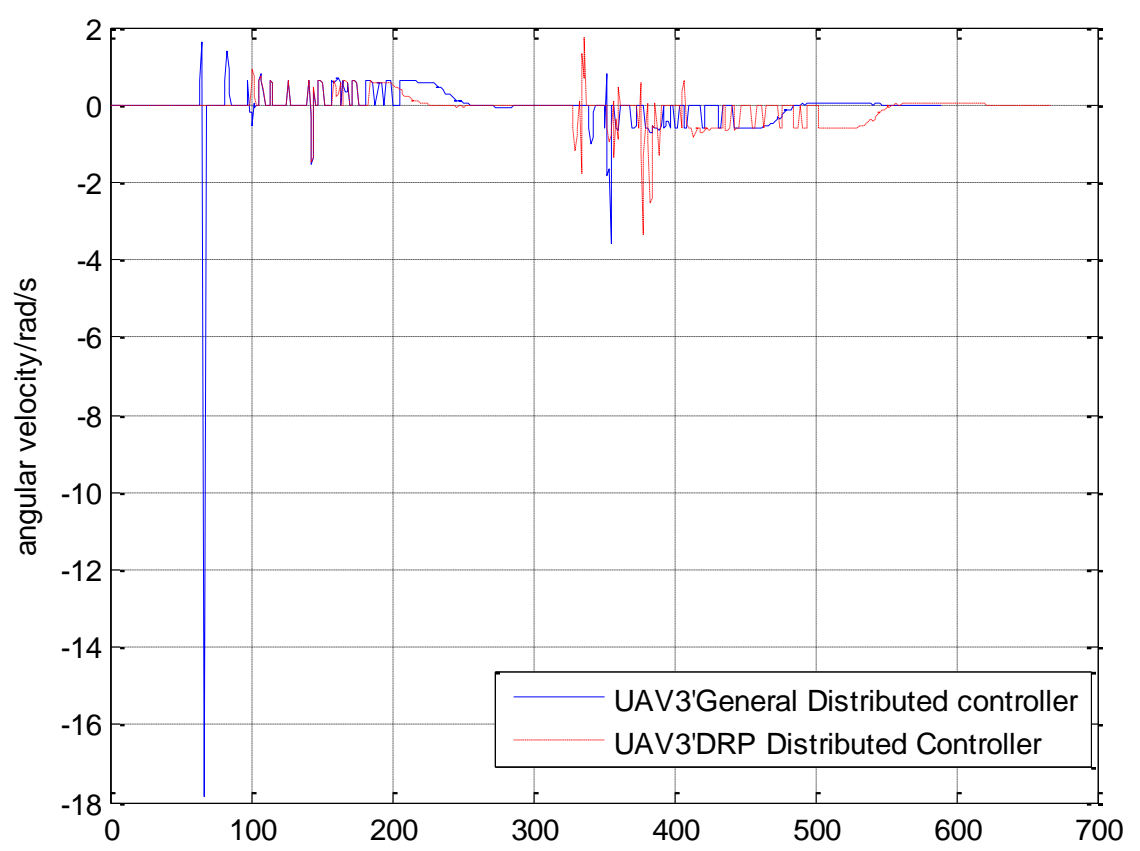

Figure 6. The Angular Velocity of UAV3 between DRP Distributed Controller and General Distributed Controller $\left(v_{d}=50 \mathrm{~m} / \mathrm{s}\right)$

From the comparative analysis of Figure 3 to Figure 6, the variations of control variant between DRP distributed controller and the general distributed controller, we can see that 
the DRP distributed controller designed in this paper has a stronger control on the formation flight in a changing situation for a good formation reconfiguration. The simulation results prove that the UAV formation using the DRP distributed steady state controller can avoid the obstacles and change back to the original formation successfully. And the accelerations and angular velocities can range in a proper and reasonable scope.

\section{Conclusions}

A multi-UAV formation is often required to change the relative positions from one to another in the task environment. In this paper, a distributed controller in terms of DRP is utilized to solve UAV formation reconfiguration control problems. With the comparison of the DRP distributed controller and the general distributed controller, the DRP distributed controller has obvious advantages over the later. Simulation results show that the new model has a better performance. The controller designed in this paper can control the wingman following the leader to maneuver and transfer from one formation to another flexibly. The relative distances between the UAVs can be adjusted and slowly approach the ideal distances when the formation is reconfigurated, so that the formation transformation will not produce maneuver mutation. This control method has an important meaning to ensure the safety of formation flight. The controller is very simple and extracts the advantages of distributed control. The trajectory characteristics are in line with the manned aircrafts. The controlled variables including acceleration in tangential and turning angular velocity in normal are small and change gently. In addition, the model established in this paper can also be used for UAV formation reconfiguration more than three UAVs.

\section{Acknowledgements}

The research work reported in this paper is supported by the National Natural Sciences Fund of China (61074159), and thanks to the researchers for the suggestions, research samples and experimental data.

\section{References}

[1] W. Q. Guo, "The Controller Design of the UAV Formation Based on Consensus Protocols", Harbin Institute of Technology, (2013).

[2] M. Pachter, J. J. Dazzo and L. Dargan, "Automatic Formation Flight Control, Control and Dynamics", vol. 6 , no. 17, (1994).

[3] F. Zhao and Y. Wei, "Close Formation Flight Control of UAVs, Aeronautical Science \& Technology", vol. 5 , no. 12, (2012).

[4] D. M. Wang and H. J. Fang, "Virtual Leaders-based Control of Flocking Motion of Intelligent Swarm", Journal Hua Zhong University of Science \& Technology, vol. 10, no. 36, (2008).

[5] J. H. Reif and H. W. Social, "A Distributed Behavioral Control for Autonomous Robots, Robotics and Autonomous Systems", vol. 6, no. 27, (1999).

[6] J. R. Lawton, R. W. Beard and B. Young, "A decentralized approach to formation maneuvers", IEEE Transactions on Robotics and Automation, vol. 6, no. 19, (2003).

[7] W. Ren, "Second-order Consensus Algorithm with Extensions to Switching Topologies and Reference Models", Proceeding of the 2007 American Control Conference; New York, America, (2007).

[8] W. W. Yu, G. R. Chen, M. Cao and J. Kurths, "Second-order Consensus for Multi-agent Systems with Directed Topologies and Nonlinear Dynamics", IEEE Trans. on Systems, Man and Cybernetics Part B: Cybernetics, vol. 3, no. 40, (2010). 


\section{Authors}

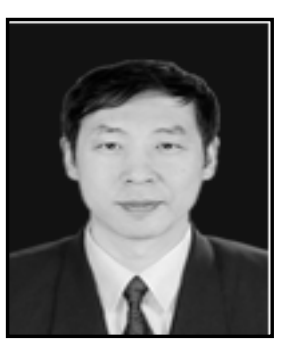

Guangyan Xu, he was born in 1964 . He is a professor in Shenyang Aerospace University. His main research interests are measurement technique, automatic control, flight control, etc..

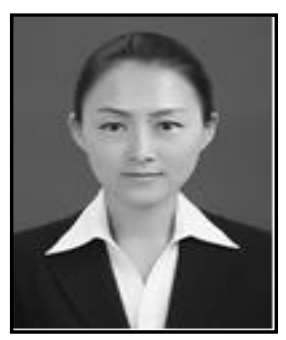

Dan Zhao, she was born in 1990. She is a graduate student in Shenyang Aerospace University. Her main research interest is unmanned aerial vehicle (UAV) formation reconfiguration.

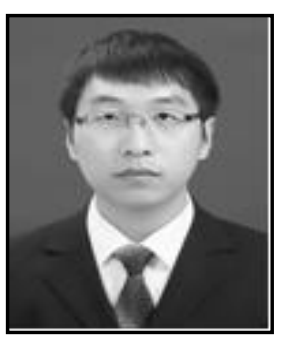

Jianchao Wang, he was born in 1988. He is a graduate student in Shenyang Aerospace University. His main research interest is unmanned aerial vehicle (UAV) formation keeping.

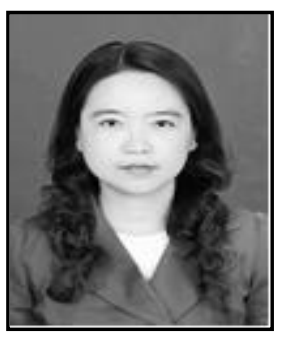

Hongmei Zhang, she was born in 1973. She is a professor in Shenyang Aerospace University. Her main research interest is optimal control theory. 
International Journal of Control and Automation

Vol. 10, No. 1 (2017) 